\title{
The Development of Basement Membranes of the Mouse Embryo ${ }^{1}$
}

\author{
G. B. Pierce" \\ With the technical assistance of Miss Susan Zajko \\ Department of Pathology, The University of Michigan, Ann Arbor, Michigan
}

Accepted December 23, 1965

\section{INTRODUCTION}

The strongest support for the concept that epithelial cells of the mouse synthesize their basement membranes is derived from the observation that three kinds of epithelia of the mouse synthesize a basement membrane in vitro in the absence of connective tissue elements (Pierce et al., 1962, 1963; Pierce, 1965). Ultrastructurally, this epithelial basement membrane (EBM) lacks the periodicity of collagen, and although it contains hydroxyproline it differs sufficiently in carbohydrate and amino acid moieties (glycine for example) to render it distinguishable from collagen (Mukerjee et al., 1965; Pierce et al., 1963). It would appear to be a distinct mucoprotein.

In a widely held concept of differentiation it is believed that cells, during morphogenesis, develop surface constitutions which render them adhesive to surfaces of complementary constitution, including those of the intercellular matrix and basement membranes (Weiss, 1950). In view of the multiplicity of cellular species, one might expect, and indeed many people feel, that basement membranes vary in their chemical composition. However, all epithelial basement membranes contain a common antigen, which might imply a degree of chemical similarity (Midgley and Pierce, 1963).

Immunohistochemical techniques have made it possible to localize

${ }^{1}$ This work was supported in part by research grants E 105 from the American Cancer Society and CA 08201, N.C.I. of the U.S.P.H.S.

${ }^{2}$ American Cancer Society Professor of Pathology. 
basement membrane and collagen with a high degree of precision (Pierce et al., 1964). A study of the development of basement membranes of the mouse embryo has been undertaken to gather information upon possible roles of this mucoprotein in morphogenesis and to obtain further biological evidence which might aid in the interpretation of its chemical nature. In view of the localization of beta globulins in renal glomeruli, which resulted in the postulate that basement membrane may be beta globulin (Tan and Kaplan, 1963), a study of the localization of beta globulins in the kidneys of fetal mice has been undertaken also.

\section{MATERIALS AND METHODS}

The methods for preparing anti-epithelial basement membrane (anti-EBM) antiserum and anti-collagen antibodies have been described in detail (Pierce et al., 1963, 1964). The anti-EBM reacted with basement membranes of all epithelia, but with neither collagen nor vascular basement membranes. Conversely anti-collagen reacted with vascular basement membranes and collagen but not with basement membranes of epithelia.

Pregnant Swiss mice were obtained from a local supplier. Advantage was taken of the fact that mice will mate within 24 hours of delivery of a litter. Accordingly female mice were separated from the males 24 hours after having littered; over $85 \%$ of these animals conceived during this 24 hours. They were sacrificed $6,7,8,9,10,11,12$, $13,14,16,18$, and 20 days after mating and, depending upon the gestational age, either whole embryos or specific organs were fixed in cold alcohol and embedded in paraffin for immunohistochemical studies according to the method of Sainte-Marie (1962).

The embryos were serially sectioned and every sixth section was stained with hematoxylin and eosin. These sections were examined microscopically, and, if suitable for the study, the adjacent unstained serial sections were allowed to react with anti-EBM or anti-collagen. The control sections were allowed to react with either normal rabbit serum or with these antibodies that had been absorbed to extinction by their respective antigens. After appropriate washing the sections were stained with fluorescein-labeled sheep antirabbit gamma globulin under the conditions described previously (Pierce et al., 1964).

For localizing beta globulins immunohistochemically in the renal 
glomeruli, rabbit antibodies to beta globulins ${ }^{3}$ characterized by gel diffusion and freed of contaminating antibodies, were employed. For immunization, each rabbit received 3 biweekly injections of $25 \mathrm{mg}$ of beta globulin in $2 \mathrm{ml}$ of complete Freund's adjuvant.

\section{RESULTS}

\section{Embryos, 6-8 Days after Fertilization}

The embryonic pole of the embryo at this stage, is composed of two cell types; a core of embryonic ectoderm from which epithelial structures and the primitive mesenchyme develop, and an overlay of proximal endoderm from which, among other structures, the primordia of the gut and respiratory apparatus will evolve. The embryo is attached to the maternal organism at the tragus but otherwise floats freely in the yolk cavity. This cavity is lined by parietal yolk sac epithelium (distal endoderm) and its basement membrane, Reichert's membrane. The latter, synthesized by the parietal yolk sac epithelium (Pierce et al., 1962, 1963, 1964), lies in apposition to the cytoplasm of trophoblastic giant cells (Figs. 1a and 2a).

When embryos of this developmental stage were allowed to react with anti-EBM, Reichert's membrane and intracytoplasmic droplets within parietal yolk sac epithelium fluoresced as had been described previously (Pierce et al., 1962, 1963), but no EBM was demonstrable in the embryo (Fig. Ib). The intracellular reaction in the parietal yolk sac epithelium presumably represented the sites of synthesis of EBM (Pierce et al., 1963, 1964). No fluorescence could be found in adjacent serial sections of these embryos stained with anti-collagen although a small amount was present in the decidua external to the trophoblastic giant cells. This nonembryonic fluorescence was diffuse in nature and lacked the fibrillar quality of collagen-reticulin (Fig. lc).

Embryos of slightly older age than the above, but still composed of only two cell types, when stained with anti-EBM had clearly visible EBM lying between ectoderm and overlying endoderm (Figs. 2a and $2 b)$. Although reacting with anti-EBM, this basement membrane failed to react with anti-collagen (Fig. 2c) and under oil emersion

${ }^{8}$ Beta globulins of the mouse were obtained from Pentex, Inc., Kankakee, Illinois. 


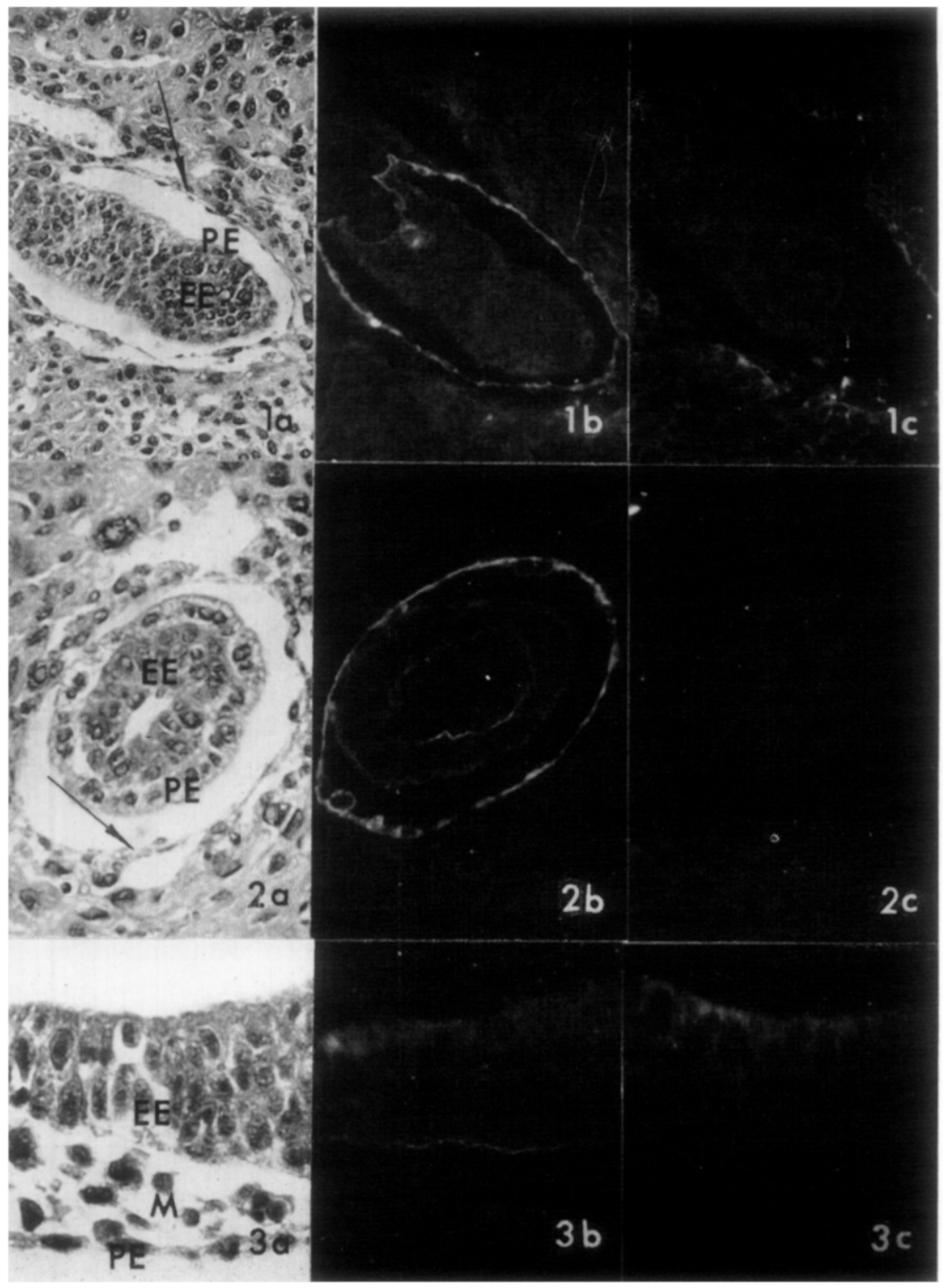

Fic. 1. Adjacent serial sections from an embryo about 6 days after fertilization, stained, respectively, with hematoxylin and eosin, anti-EBM, and anticollagen. (a) The relationship of embryonic ectoderm (EE) and proximal endoderm $(P E)$, which together form the embryo proper, is illustrated. Parietal yolk 
appeared to be a single lamina intimately associated with the embryonic ectoderm.

At a slightly later stage of development when the embryo had formed a neural groove with a layer of mesenchyme occupying the angle formed between the neuroectoderm and endoderm, delicate lamellae of EBM followed the contours of the endodermal and ectodermal layers (Figs. $3 \mathrm{a}$ and $3 \mathrm{~b}$ ). It was inferred from this observation that both ecto- and endoderm were synthesizing basement membrane antigens. Neither the newly formed mesenchymal cells nor the basement membrane reacted with anti-collagen (Fig. 3c).

\section{Embryos, 9-10 Days after Fertilization}

The neural tube was completely ensheathed by a layer of EBM which appeared to be the embryonic counterpart of the pia-glial membrane of the adult. This layer was well developed in comparison to some of the other basement membranes of the embryo. For instance, the basement membrane of the skin was more difficult to refract than that of the neural tube; it was often so thin that it appeared discontinuous. In areas where the neural groove still persisted the basement membrane of the neural apparatus and that of the skin were continuous (Figs. $4 \mathrm{a}$ and $4 \mathrm{~b}$ ) as has been described histochemically by Chiquoine (1957). Continuity was also observed be-

sac epithelium and Reichert's membrane (arrow) may be seen in the extraembryonic area. (b) Note the absence of EBM in the embryo proper. Reichert's membrane contains EBM. (c) Collagen is not present in the embryo. Magnification: $\times 150$.

Fig. 2. Adjacent serial sections from an embryo about $6 \frac{1 / 2}{2}$ days after fertilization, stained, respectively, with hematoxylin and eosin, anti-EBM, and anticollagen. The plane of sectioning is at right angles to that of Fig. 1. ( $a$ and b) Note the relationship of embryonic ectoderm $(E E)$ and proximal endoderm $(P E)$. An epithelial basement membrane lies between them. Reichert's membrane and parietal yolk sac epithelium (arrow) are also stained. (c) Ihere is an absence of collagen. This section also serves as a control for Fig. $2 b$ and indicates the immunological specificity of the EBM-anti EBM reaction. Magnification: $\times 290$.

FIG. 3. Adjacent serial sections of an embryo stained with hematoxylin and eosin, anti-EBM, and anti-collagen, respectively. (a) Mesenchyme ( $M$ ) separates embryonic ectoderm $(E E)$ and proximal endoderm $(P E)$. (b) Delicate lamellae of epithelial basement membrane lie adjacent to ectoderm and endoderm. (c) Collagen was not demonstrable in this embryo. This section also serves as a negative control for Fig. $3 \mathrm{~b}$. Magnification: $\times 490$. 


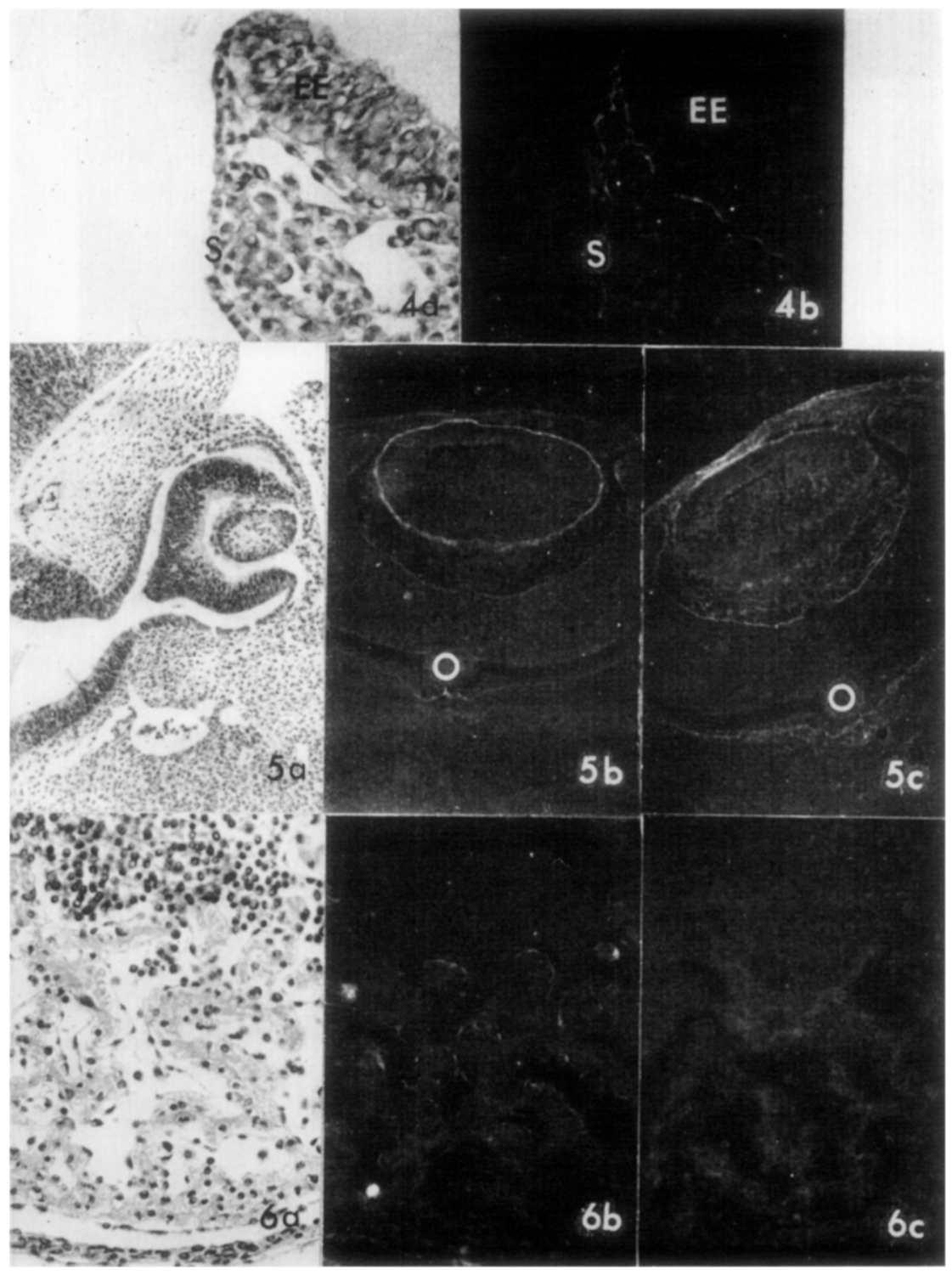

Frg. 4. ( $\mathrm{a}$ and $\mathrm{b}$ ) Sections stained, respectively, with hematoxylin and eosin and anti-EBM, from the tail region of the same embryo, but they are not adjacent serial sections. The continuity of the basement membrane of the embryonic epithelium ( $E E$ ) of the neural groove and of the skin (S) is illustrated. Magnification: $\times 400$. 
tween the basement membranes of the skin and lens capsule, and the optic cup and neural apparatus via the optic nerve. Since the eye develops as an outgrowth of the central nervous system, it was not surprising to find the optic nerve and optic cup invested by the same EBM (Figs. 5a, 5b, and 5c). These observations were in agreement with the ultrastructural findings of Cohen (1961).

EBM of the optic cup was exquisitely delicate and difficult to refract (Figs. $5 \mathrm{~b}$ and $5 \mathrm{c}$ ), but that of the lens capsule was easily visible. In the earliest stages of lens differentiation, in confirmation of Cohen (1961), the basement membrane of the lens primordium was continuous with that of the skin. Later, after the lens had separated from the epidermis, it remained associated with EBM which was synthesized presumably by lens epithelium (Figs. $5 \mathrm{~b}$ and $5 \mathrm{~d}$ ). Collagen, or material stainable with anti-collagen, was present in the space between the lens and the retinal primurdia (Fig. 5c), however the lens capsule did not react with anti-collagen.

Material reacting with anti-collagen was observed in the aortas and hearts of these embryos. In the aorta this fluorescence was in the form of threadlike strands which were presumed to be collagen-reticulin. At about the same time the endocardium stained specifically with anti-collagen. This reaction formed a membranous band which appeared to be in the endothelial basement membrane and in the cytoplasm of the endothelial cells (Figs. $6 \mathrm{a}, 6 \mathrm{~b}$, and $6 \mathrm{c}$ ). The sarcolemma

Fic. 5. (a) Section of the eye of a mouse embryo of 9-10 days' gestation stained with hematoxylin and eosin to illustrate the continuity of optic cup and neuroectoderm and the relationship of lens to skin. At this low magnification $(\times 50)$, it was impossible to demonstrate the continuity of the basement membrane of the optic cup and neuroectoderm with the fluorescent antibody technique. ( $b$ and $c$ ) These are adjacent serial sections near the optic nerve $(O)$ of an eye stained with anti-EBM and a mixture of anti-EBM and anticollagen, respectively. In (b) only the basement membrane is stained whereas in (c) basement membrane and collagen are stained. (b) There is continuity of a delicate lamella of EBM over the optic cup and small portion of the optic nerve. The lens capsule reacts strongly with anti-EBM. (c) In addition to the faint staining of the basement membrane of the optic cup, collagen can be localized between the posterior surface of the lens and optic cup and adjacent to the basement membrane continuous with the optic nerve. Magnification: $\times 96$.

FIG. 6. (a-c) Sections from the same heart stained, respectively, with hematoxylin and eosin, anti-collagen, and normal rabbit serum. (a) This section illustrates the relationship of endothelium and myocardium. (b) There is positive staining of endothelial basement membranes, but sarcolemma of cardiac muscle is not stained. (c) This control is negative. Magnification: $\times 96$. 


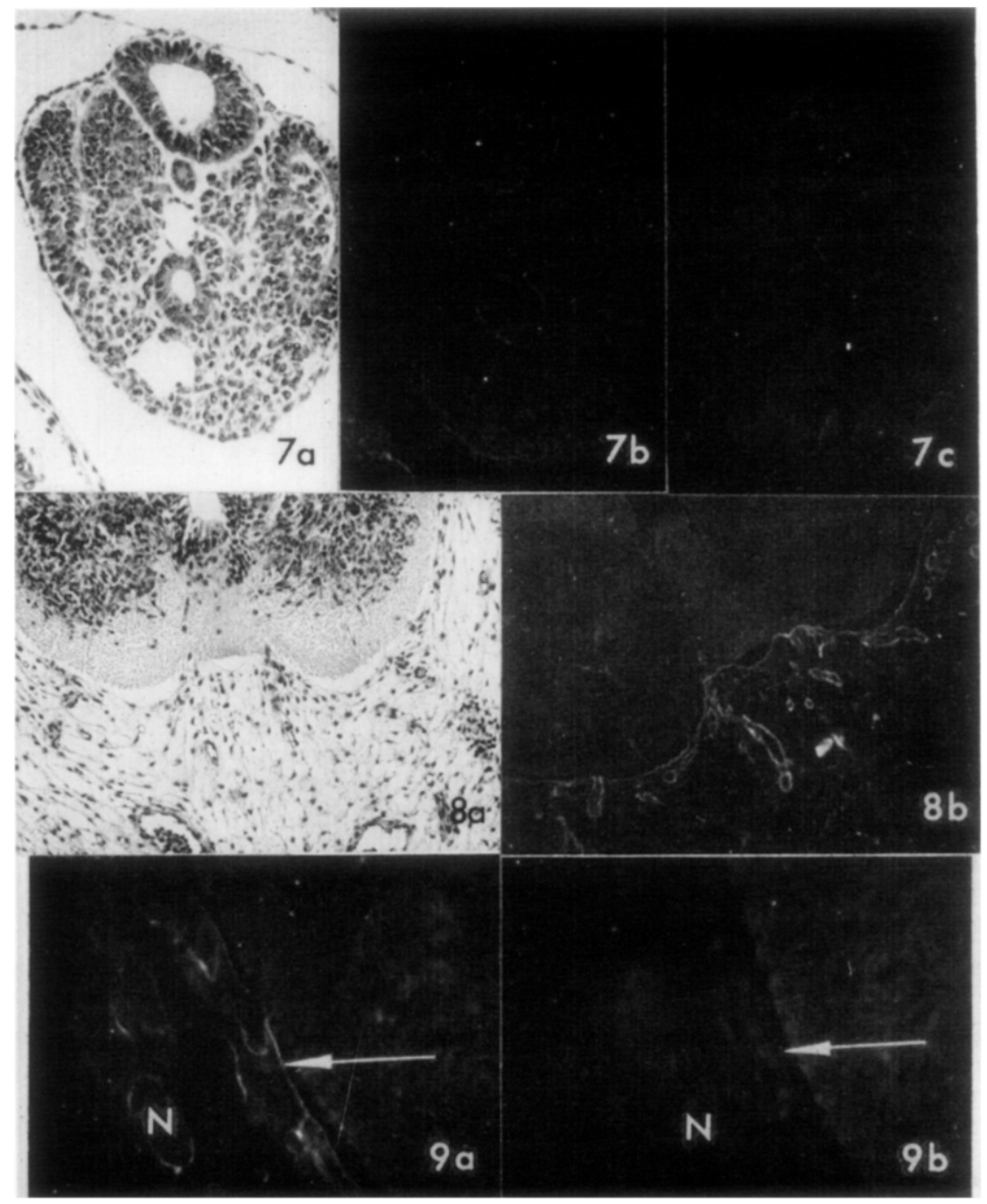

FIG. 7. (a-c) Adjacent serial sections from the tail region of a 10- to 11-day embryo stained, respectively, with hematoxylin and eosin, anti-EBM, and antiEBM absorbed with EBM. (a and b) The neural tube, gastrointestinal tube, and skin are each associated with an epithelial basement membrane. There is continuity of basement membranes of the neural tube and skin. (c) The control is negative. Magnification: $\times 154$.

FIG. 8. ( $a$ and b) Sections from a 12-day embryo stained with hematoxylin and eosin and a mixture of anti-EBM and anti-collagen, respectively. Note the 
of the muscle fibrils of the ventricles did not stain with anti-collagen nor did the mesenchymal elements surrounding the primordium of the lungs. Fibrils reacting faintly with anti-collagen were present in the mesenchyme around the epithelium of the gastrointestinal tube and in the skin.

\section{Embryos, 10-11 Days after Fertilization}

EBM ensheathed most of the epithelial structures present at this stage of development (Figs. $7 \mathrm{a}, 7 \mathrm{~b}$, and $7 \mathrm{c}$ ). Basement membranes of one organ system were still continuous with those of another. For instance, those of the skin and spinal cord in the region of the tail werc continuous as were those of the ncural tubc and eye.

Fibrils, stainable with anti-collagen, were present around the epithelium of the intestine, and in the lung, liver, and spleen. Precartilage of the vertebrae failed to react with anti-EBM but reacted specifically with anti-collagen as very fine extracellular fibrils. When the matrix of mature collagen was laid down, this reaction was lost, suggesting that the antigenic sites might have been masked by the macromolecules of ground substance. The basement membrane of the notochord did not react with anti-EBM but stained brilliantly with anti-collagen. The somites were outlined with fine fibrils that reacted with anti-collagen, but the cells of the somites reacted with neither anti-collagen nor anti-EBM.

\section{Embryos, 11-12 Days after Fertilization}

The external limiting membrane of the neural tube stained readily with anti-EBM. Instead of appearing as a smooth delicate membrane as it had at earlier stages, numerous saccules were observed in it (Figs. 8a and b). These sacculcs wcre not apparent in the specimens stained with hematoxylin and eosin (Fig. 8a). They surrounded cells which appeared to be migrating away from the neural epithelium.

numerous outpouchings in the external limiting membrane, which are not as readily apparent in the routine histologic preparation. $\times 122$.

FIG. 9. ( $a$ and b) Adjacent serial sections to illustrate the basement membranes of the neural crest and neural tube. They were stained, respectively, with anti-EBM and normal rabbit serum. (a) The external limiting membrane (arrow) and basement membrane surrounding neural crest cells $(N)$ contain EBM. Other cellular aggregates which appear to be budding from the neural tube and which may be small vessels are also associated with EBM. (b) The control is negative. Magnification: $\times 320$. 


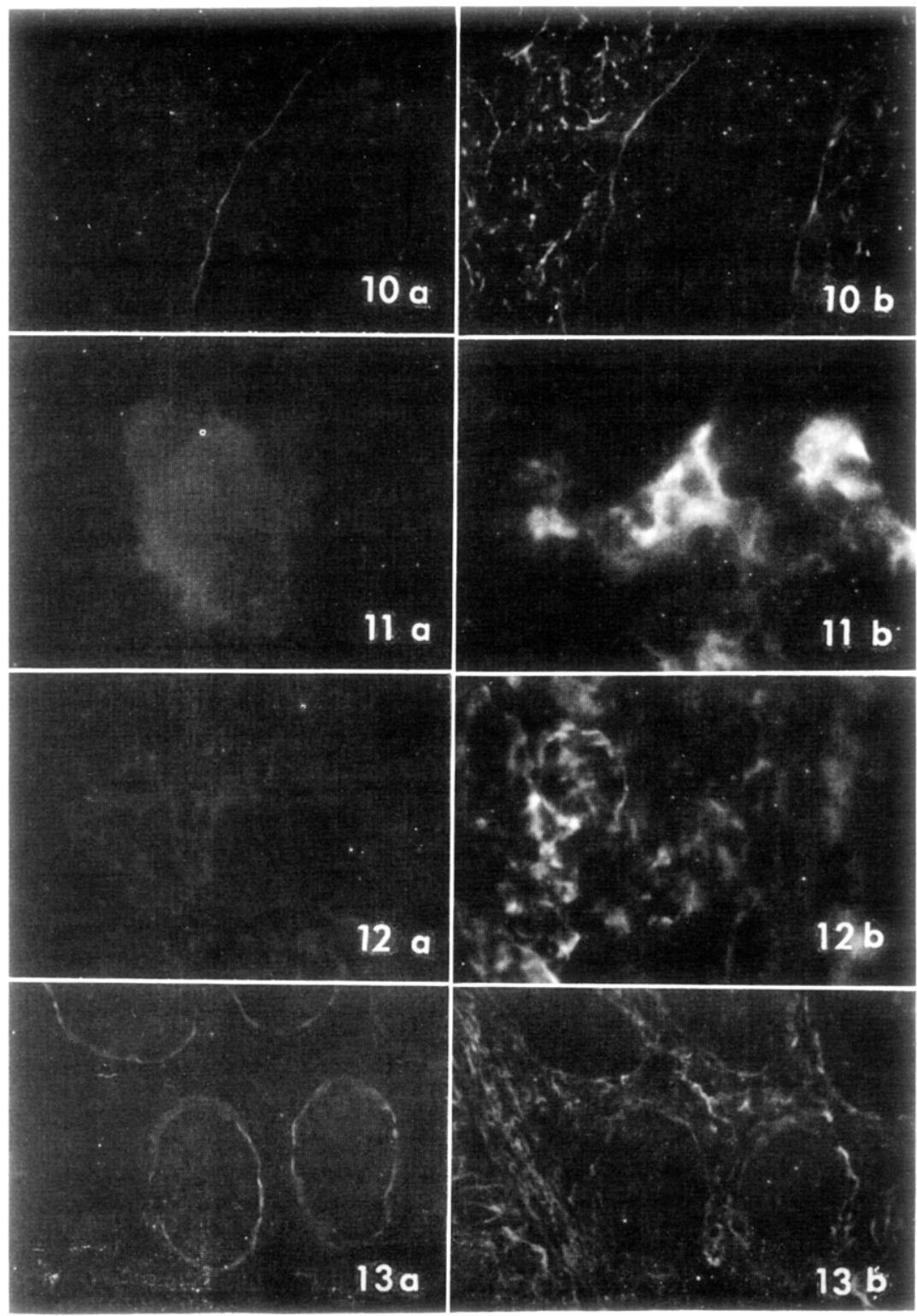

Fig. 10. ( $a$ and b) Adjacent serial sections of the gastrointestinal tube stained with anti-EBM and anti-collagen, respectively. (a) Note the epithelial basement membrane and the inability of anti-EBM to react with collagen and 
Dorsally, these migrating cells appeared to be forming the neural crest, but others on the lateral and ventral surfaces were apparently differentiating into other structures (Figs. 9b, 10a, and 10b). Some of these migrating cells may be Schwann cells as described by Raven (1937) or they may be satellite cells (Brizzee, 1949). In the adult, EBM is not associated with Schwann cells, although these cells have a basement membrane visible electron microscopically. This basement membrane reacts with anti-collagen in a manner similar to that of muscle cells or fat cells (Midgley and Pierce, 1963). What appeared to be small blood vessels in the region ventral to the neural tube contained EBM (Figs. 9a, 9b). This was not an artifact because the same epithelial specific anti-EBM failed to stain reticulin or vascular basement membranes in other areas of the same section (Figs. 10a and $10 \mathrm{~b}$ ), and suggests that blood vessels in this region may differentiate from cells of the neural tube that can synthesize EBM. In later stages of development the vessels in the region of the central nervous system failed to react with anti-EBM. Neither membranes nor cells within the central nervous system contained EBM.

Unlike the situation in the adult, the bronchi of the embryo were invested by epithelial basement membrane but the reaction was never intense. Likewise, the biliary tree of the embryo which buds from the primitive gastrointestinal tube had demonstrable EBM, but in the adult EBM was absent from hepatic cords (Midgley and Pierce, 1963). Whether or not this is an adaptive change to facilitate transport of macromolecules is not known.

In later stages of development the basement membranes seemed to vary little from the description given above. Those of the lung

vascular basement membrancs. (b) The mesenchymal reactions of anti-collagen are illustrated. Magnification: $\times 490$.

FIG. 11. ( $a$ and $b$ ) Sections illustrating the equivocal reaction of anti-beta globulin with EBM of the parietal yolk sac carcinoma in comparison to the intense reaction of this reagent with the glomerulus of an adult mouse. Magnification: $\times 490$.

Fic. 12. ( $a$ and b) Adjacent serial sections from the kidney of a mouse of 17 days' gestation reacted with anti-beta globulin and anti-EBM, respectively. (a) Anti-beta globulin fails to react with the kidney. (b) EBM was present in primitive glomeruli and tubules. Magnification: $\times 290$.

FIg. 13. ( $a$ and b) Adjacent serial sections of testis of a fetal mouse 17 days after fertilization stained with anti-EBM and anti-collagen. (a) Note the thick epithelial basement membrane of the tubules. (b) The stroma stains with anti-collagen. Magnification: $\times 260$. 
seemed to become reduced in thickness as development progressed. After expansion of alveoli it was impossible to demonstrate this antigen in the lungs or even around the bronchi. Neither the reason for this loss nor the mechanism responsible was apparent.

EBM was present in the renal primordia long before glomeruli had differentiated: each primordial tubule was surrounded by a rather poorly defined lamella. Abundant material stainable with anti-collagen was present in the basement membranes of the numerous capillaries. Where capillary and tubule made contact these antigenically distinct basement membranes appeared to fuse and to form a single basement membrane as has been demonstrated ultrastructurally using ferritinlabcled anti-EBM and ferritin-labcled anti-collagen (Pierce et al., 1964).

EBM and vascular basement membrane antigens were present in the delicate basement membranes of developing glomeruli, but the relative positions of these antigens has not been determined directly. In view of the observations and deductions of Kurtz (1958) and our demonstration of the dual antigenic nature of tubular basement membranes, it would seem reasonable to assume that these antigens lie in juxtaposition. Each persists in the glomerulus throughout the life of the animal.

Beta globulins were present in the basement membranes of glomeruli and tubules of the adult mouse, but the reaction of anti-beta globulin in EBM of a parietal yolk sac carcinoma was barely discernible (Figs. 1la and 1lb). Since the EBM of the tumor is not involved in filtration as is that of the kidney, it was concluded that beta globulin might be present in the normal basement membranes as a result of adsorption during filtration. In support of this idea was the observation that fetal glomeruli that had minimal opportunity for filtration contained EBM but no beta globulin by immunofluorescence (Figs. 12a and 12b). Furthermore, fetuses of 16-18 days gestation were homogenized in an equal volume of phosphate-buffered saline in the cold, and after clearing by centrifugation the extract was allowed to react with anti-beta globulin in gel diffusion technique. No precipitin lines developed although they were observed between diluted adult mouse serum and anti-beta globulin. Accordingly, it was concluded that little if any beta globulin was present in the fetus at this time and that beta globulin was not an integral part of the basement membrane but was adsorbed to it during filtration.

EBM developed early in the testis and appeared as a dense band 
around the tubules (Figs. 13a and $13 \mathrm{~b}$ ). In the ovary the antigen was observed around primitive sex cords and later around the follicles. The breast primordium which develops from the skin as a bud was invested by EBM which was continuous with that of the skin. In later development the mammary ducts were always associated with epithelial basement membrane; during pregnancy, the alveoli were invested by it and during involution it was removed, presumably by enzymatic action (Pierce, unpublished).

\section{DISCUSSION}

The epithelial basement membrane antigen is one of the first extraccllular macromolecules synthesized in the mouse embryo. Shortly after endodermal differentiation an epithelial basement membrane appears between embryonic ectoderm and the newly formed endoderm. Since endoderm appeared before the basement membrane, it follows that the latter did not play a role in the induction of the former. If this single example is typical of differentiation in general, then basement membranes would not appear to play a direct role in the process of induction. Grobstein and Dalton (1957) demonstrated that inductive phenomena, whatever their nature, can be mediated across filters that exclude cell contact. It is not surprising then that differentiations such as that of the lens from ectoderm take place in in vivo situations in which the inductor (optic cup) is on one side of a basement membrane and the competent responders (ectoderm) are on the other.

It was impossible to determine the mode of formation of the first basement membrane. Although embryonic ectoderm and endoderm may have each contributed to it, in an ultrastructural study (unpublished), the basement membrane followed the contours of the former, which suggested ectoderm as the origin. The idea that more than one cell type can contribute to a common basement membrane was developed by Kurtz (1958), who observed that the human renal glomerular basement membrane was formed by fusion of lamellae associated with each of endothelium and glomerular visceral epithelium.

Many differentiated tissues develop as outgrowths from a primordium, and in the early stages of this process the developing bud shares a common basement membrane with its progenitor. After further development with migration away from the parent tissue the newly differentiated tissue continues to synthesize epithelial basement membrane. The development of the optic cup and lens are examples of this phenomenon. In view of this mode of development and the persis- 
tency without modification of the epithelial basement membrane antigen throughout the life of the animal it would seem reasonable to expect that basement membranes of epithelia would be similar in chemical structure. Direct proof for this speculation will have to await the isolation and chemical analysis of basement membrane mucoprotein free of tissue stroma which has been considered an integral part of it.

At this point it would be desirable to clarify terminology. The basement membrane of the amphibian skin is composed of an adepidermal membrane plus many layers of ordered collagen. The basement membrane of the mammalian skin is composed of an amorphous band of relatively electron dense material that appears to correspond to the adepidermal membrane of the amphibian. Adjacent to it is the disordered collagen and elastic tissue of the dermis. The epithelial basement membrane of the mammal is synthesized by the adjacent epithelial cell (Pierce, 1965). The collagen fibrils in juxtaposition to it need not be considered an integral part of the epithelial basement membrane because Reichert's membrane and the basement membrane of the embryonic epithelium are synthesized in situations devoid of collagen.

The unavoidable inclusion of collagen in preparations of basement membrane for chemical or immunological analysis leads to gross errors. Likewise, it has been impossible for us to obtain a preparation of lens capsule free of lens fibrils. The epithelial basement membrane employed in our studies was obtained from the ascites form of a tumor so that contamination of any kind was minimal (Pierce et al., 1964). Since it is of neoplastic origin, the remote possibility exists that it may differ chemically from its normal counterpart.

The chemcal naturc of bascment membrane has not been determined. Analysis of epithelial basement membrane of neoplastic origin indicates that it contains almost as much hydroxyproline as collagen but only half of the glycine. In addition, the carbohydrate moieties differ from collagen (Mukerjee et al., 1965). Accordingly, it has been considered as a mucoprotein distinct from collagen. On the other hand, the data do not rule out the possibility that the epithelial basement membrane may be composed of a tropocollagen core to which is bound an additional antigenic mucoprotein synthesized by epithelium. Although data are not available to settle the issue some facts are relevant. 
The observation that the embryo synthesizes Reichert's membrane, and the basement membrane between ectoderm and endoderm, in the absence of connective tissues, indicates that if tropocollagen is part of the basement membrane molecule it too must be synthesized by the epithelium. We have been unable to localize this antigen immunohistochemically in epithelial basement membrane, but it may have been masked by other components. Moreover, it has not been possible to localize this antigen within epithelial cells synthesizing the epithelial basement membrane.

Hay and Revel (1963), on the basis of turnover of labeled proline in amphibians, consider the epithelium to be synthesizing a collagenous material which is incorporated in the adepidermal membrane. If content of hydroxyproline is the sole criterion for identification of collagen, then this basement membrane by definition would be collagen. Others have analyzed glomerular basement membrane using highly sophisticated techniques and have concluded that it is collagen (Lazarow and Speidel, 1964). As described above, glomerular basement membrane is antigenically inhomogeneous and contains in addition to the epithelial basement membrane a distinct antigen that cross reacts with vascular basement membrane. In addition, chemical analysis of glomerular basement membrane from disease states such as diabetes will reflect the develoment of new collagen from the degenerative and repair processes that characterize the renal manifestations of this disease. Until the inherent inhomogeneity of glomerular basement membrane is described in chemical terms and the likely contamination of the material by collagen of the repair process has been excluded, little significance can be read into the chemical analysis.

Although antigenic dissimilarities have been shown to exist between epithelial basement membrane and collagen, the possibility exists that there may be antigenic similarities. Epithelial basement membrane has always been contaminated by trace amounts of collagen so it has been impossible to determine whether basement membrane and collagen possess common antigens. If the latter proves to be true then a strong argument will be at hand for considering epithelial basement membrane as a unique type of collagen.

Tan and Kaplan (1963) have reported the localization of beta globulins in the glomerular basement membranes of mice and have postulated that basement membranes may be beta globulin. On the other hand, we have proposed that beta globulins are adsorbed to the 
basement membrane during filtration and are not an integral part of it (Pierce et al., 1964). Since beta globulins contain serum enzymes, adsorption of these enzymes to the basement membrane may represent the means of cleaning this filter. We have confirmed the localization of beta globulins in basement membranes of the adult but not in the fetus, where minimal filtration would have occurred. Thus it would appear that localization of beta globulins is a function of filtration and does not represent a structural part of the basement membrane.

Neither the stimulus for basement membrane synthesis nor the function of basement membranes was apparent from this study. Reichert's membrane and the basement membranes of the embryonic ectoderm develop between epithelial and/or endodermal layers; this would suggest that connective tissue is not the stimulus for the synthesis of basement membrane. In epithelial tumors the basement membrane was usually at the tumor stromal junction as in the normal situation. However, in adenocarcinomas with glandular acini, basement membrane invested acini and was itself invested by other malignant epithelial cells (Pierce, 1965). This might suggest that the stimulus for the formation of basement membrane is mediated at the tissue level by the differentiating epithelium in response to the needs of the epithelium.

As for the function of basement membranes, pathologists have considered the glomerular basement membrane as a filter, which in glomerulonephritis becomes thickened and permeable to serum protein. In nephritis, much of the apparent thickening of basement membranes visible light microscopically is due to the deposition of antigen-antibody complexes which are biologically active and cause dysfunction (Dixon et al., 1961). Whether or not the basement membrane is the renal filtcr capable of retaining serum protein under normal circumstances has never been demonstrated. Molecules of the size of ferritin are trapped within its substance (Farquhar et al., 1961), but these molecules, of diameters of 110-120 \&, are larger than the theoretical pore size postulated to be necessary for retention of serum protein (Pappenheimer, 1953).

It is conceivable that basement membrane might serve as the catalyst for polymerization of tropocollagen into collagen fibrils, an idea first suggested by Weiss and Ferris (1956) and critically discussed by Edds (1964). The experiments of Hay and Revel (1963) 
indicated that newly formed collagen polymerized adjacent to the adepidermal membrane of the skin of the salamander. It is also conceivable that in regions remote from epithelial basement membranes, vascular basement membranes could serve this function. Recently, in an in vitro system in which epithelium was grown on one side of a Millipore filter and mesenchyme on the other, Kallman and Grobstein (1965) demonstrated the polymerization of tropocollagen into collagen on the epithelium. Apparently, tropocollagen synthesized by the mesenchyme diffused across the Millipore filter and polymerized into collagen on the surface of the epithelium. The role, if any, of epithelium itself or the epithelial basement membrane in initiating this polymerization is not known.

\section{SUMMARY}

The development of collagen and epithelial basement membranes was studied immunohistochemically in mouse embryos utilizing specific antibodies to collagen that would not react with basement membranes of epithelia and antibodies to epithelial basement membranes that would not react with collagen.

An epithelial basement membrane appeared prior to the development of mesenchyme in embryos of 5-6 days of age and lay between embryonic epithelium and proximal endoderm. Collagen which was not present in the embryo at this stage appeared later; it was observed first in the walls of the aorta.

A differentiating epithelial organ usually develops as a bud from a primordium and shares a common basement membrane with its progenitor. The epithelial basement membrane antigen is associated with the newly developed tissue after detachment from the parent tissue and subsequently for the life of the animal.

The data are in accord with the previously established concept that epithelia synthesize their basement membranes and in addition they suggest the probability that epithelial basement membranes are chemically similar.

\section{REFERENCES}

BrizzeE, K. R. (1949). Histogenesis of the supporting tissue in the spinal and the sympathetic trunk ganglia in the chick. J. Comp. Neurol. 91, 129-146.

Culquorne, D. (1957). The distribution of polysaccharides during gastrulation and embryogenesis in the mouse embryo. Anat. Record 129, 495-516. 
CoHes, A. I. (1961). Electron microscopic observations of the developing mou eye. I. Basement membranes during early development and lens formatio Develop. Biol. 3, 297-316.

Dixon, F. J., Feldman, J. D., and Vazquez, J. J. (1961). Experiment glomerulonephritis. The pathogenesis of a laboratory model resembling $t$ spectrum of human glomerulonephritis. J. Exptl. Med. 113, 899-920.

EDDs, M. V. (1964). The basement lamella of developing amphibian skin. "Small Blood Vessel Involvement in Diabetes Mellitus" (M. D. Siperstej A. R. Colwell, Sr., and K. Meyer, eds.), pp. 245-250. Amer. Inst. Biol. Sc Washington, D.C.

Fanquhah, M. G., Wissig, S. L., and Palade, G. E. (1961). Glomerular perm ability. I. Ferritin transfer across the normal glomerular capillary wall. Exptl. Med. 113, 47-66.

Grobstein, C., and Dalton, A. J. (1957). Kidney tubule induction in mou metanephrogenic mesenchyme without cytoplasmic content. J. Exptl. Zo $135,57-73$.

Hay, E. D., and Revel, J. P. (1963). Autoradiographic studies of the origin the basement lamella in Ambystoma. Develop. Biol. 7, 152-168.

Kallman, F., and Grobstein, C. (1965). Source of collagen at epitheliomese chymal interfaces during inductive interaction. Develop. Biol. 11, 169-183.

Kuntz, S. M. (1958). The elcctron microscopy of the developing human rer glomerulus. Exptl. Cell Res. 14, 355-367.

Lazarow, A., and Speidel, E. (1964). The chemical composition of the g] merular basement membrane and its relationship to the production of diabe complications. In "Small Blood Vessel Involvement in Diabetes Mellitu (M. D. Siperstein, A. R. Colwell, Sr., and K. Meyer, eds.), pp. 127-1E Amer. Inst. Biol. Sci., Washington, D.C.

Midgley, A. R., JR., and Pience, G. B., JR. (1963). Immunohistochemical an: ysis of basement membranes of the mouse. Am. J. Pathol. 43, 929-943.

Mukerjee, H., Sri Ram, J., and Pierce, G. B. Jr. (1965). Basement membran V. Chemical composition of neoplastic basement membrane mucoprotein. A J. Pathol. 46, 49-58.

Pappenheimer, J. R. (1953). Passage of molecules through capillary wa] Physiol. Rev. 33, 387-423.

Pierce, G. B., JR. (1965). Basement membranes. V1. Synthesis by epithel tumors of the mouse. Cancer Res. 25, 656-670.

Pierce, G. B., Jr., Midgley, A. R., Jr., Sri Ram, J., and Feldman, J. D. (1962 Parietal yolk sac carcinoma: Clue to the histogenesis of Reichert's membranc the mouse embryo. Am. J. Pathol. 41, 549-566.

Pierce, G. B., Jr., Midgley, A. R., Jr., and Sri Ram, J. (1963). The hisi genesis of basement membranes. J. Exptl. Med. 117, 339-348.

Pierce, G. B., Beals, T. F., Sri Ram, J, and Midgley, A. R. (1964). Baseme Parietal yolk sac carcinoma: Clue tothe histogenesis of Reichert's membrane Pathol. 45, 929-962.

Raven, C. P. (1937). Experiments upon the origin of sheath cells and symf thetic neuroblasts in amphibia. J. Comp. Neurol. 67, 221-240. 
SaINTE-Marie, G. (1962). A paraffin embedding technique for studies employing immunofluorescence. J. Histo. Cytochem. 10, 250-256.

TAN, E. M., and KAPLAN, M. H. (1963). Immunological relation of basement membrane and a serum beta globulin in the mouse. Demonstration of renal basement membrane alteration in mice injected with Streptolysin S. Immunology 6, 331-344.

WEIss, P. (1950). Perspectives in field of morphogenesis. Quart. Rev. Biol. 25, $177-198$.

Weiss, P., and Ferrus, W. (1956). The basement lamella of amphibian skin. Its reconstruction after wounding. J. Biophys. Biochem. Cytol. 2, 275-281, Suppl. 